Supplement of The Cryosphere, 14, 1187-1207, 2020

https://doi.org/10.5194/tc-14-1187-2020-supplement

(c) Author(s) 2020. This work is distributed under

the Creative Commons Attribution 4.0 License.

(c) (1)

The Cryosphere

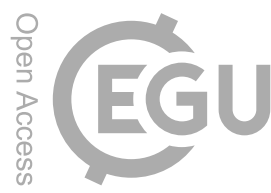

Supplement of

\title{
How useful is snow accumulation in reconstructing surface air temperature in Antarctica? A study combining ice core records and climate models
}

Quentin Dalaiden et al.

Correspondence to: Quentin Dalaiden (quentin.dalaiden@uclouvain.be)

The copyright of individual parts of the supplement might differ from the CC BY 4.0 License. 


\section{S1: Statistical surface air temperature reconstructions from Stenni et al. (2017)}

Using the $\delta^{18} \mathrm{O}$ composites, Stenni et al. (2017) reconstructed regional surface air temperature over the last two millennia based on the statistical relationship between $\delta^{18} \mathrm{O}$ and surface air temperature. Three methods have been used to scale the $\delta^{18} \mathrm{O}$ composites. In the first approach, the regional slopes between $\delta^{18} \mathrm{O}$ and temperatures were computed from the outputs

5 of the ECHAM5-wiso model forced by ERA-Interim atmospheric reanalysis (Goursaud et al., 2018) over the 1979-2013 period. In the second method, the reconstruction obtained from the first method for the WAIS region is corrected using an independent temperature record: the borehole temperature reconstruction at WAIS divide (Orsi et al., 2012). This allows to match the cooling trend over the 1000-1600 period (Stenni et al., 2017). This method provides a different reconstruction for the WAIS region - implying thus also the West Antarctic and the whole Antarctic reconstructions -, but not for the regions in East Antarctica. Finally, in the third method, the regional normalized $\delta^{18} \mathrm{O}$ composites have been scaled to the variance of the surface air temperature observations (e.g. Nicolas and Bromwich, 2014) over the 1960-1990 period. The second reconstruction is used throughout this study for two reasons: 1) the third method is based on the surface air temperature observations, which are used here to estimate the skill of the reconstructions which could lead to a bias; 2) the correction introduced in the second method is expected to improve the reconstruction compared to the previous method. The temporal resolution of these surface air temperature reconstruction is the same as the $\delta^{18} \mathrm{O}$ composites: 10 years for $0-1800$ period and 5 years for 1800-2010 period.

\section{S2: Defining uncertainties associated with proxy data used during data assimilation process}

Data assimilation requires estimates of the uncertainty associated with the proxy data used. Unfortunately, uncertainty estimations are not provided with published reconstructions used here and the instrumental time series are too short to reliably derive the uncertainty. If we apply the same error for all the Antarctic regions, the assimilation will tend to give more weight to the time series that have more variance (i.e. the high-accumulation regions). On the other hand, if we apply an error proportional to the standard deviation of the time series, each region will tend to have the same weight. The uncertainly could also be related to the amount of ice cores included in each regional composite, but the link between this number and the quality of the composite is not straightforward (Stenni et al., 2017). Several experiments have been performed to test the impact of different estimates of the data uncertainties on the data assimilation results. The results are qualitatively similar to standard choices of the uncertainty (Klein et al., 2019). The experiments shown here assume a signal to noise ratio of 1 for each regional composite. This is probably an optimistic estimate but this has the advantage of providing a strong data constraint and the comparison of the reconstruction using data assimilation with instrumental data indicates a good skill of the methods using this value.

\section{S3: Present-day AIS SMB simulated by GCMs}

Overall, the AIS SMB simulated by GCMs is in good agreement with the SMB simulated by the regional climate model RACMO2 over the last decades (1979-2005, R² = 0.63; Figs. S5, S6, S7 and S8). As expected, both display high values of 
SMB along the coast ( $>300 \mathrm{~mm}$ w.e. year ${ }^{-1}$ ) - especially for West Antarctica and the Antarctic Peninsula - and lower values at high elevations (e.g. the Plateau: $<100 \mathrm{~mm}$ w.e. year $^{-1}$ ). The mean of the SMB over the entire AIS simulated by the selected models (including isotope-enable models) is $6.4 \mathrm{~mm}$ w.e. year ${ }^{-1}$ lower than the SMB simulated by RACMO2 over the 19792005 period (relative bias: -3.4\%; Fig. S8 for the correlation plots for each model and Fig. S9 for the integrated SMB over the 5 entire AIS for each model).

However, Figure S5 shows that the GCMs, compared to RACMO2, underestimate SMB in areas below $1500 \mathrm{~m}$ (mean bias of $-55 \mathrm{~mm}$ w.e. year ${ }^{-1}$; relative bias: $-15 \%$ ) over 1979-2005. For the areas above $1500 \mathrm{~m}$, the mean bias of the simulated SMB by GCMs compared to RACMO2 is $11 \mathrm{~mm}$ w.e. year ${ }^{-1}$ (relative bias: $11 \%$ ). These results are in agreement with previous studies (e.g. Palerme et al., 2017; Genthon et al., 2009; Krinner et al., 2008) who have shown that due to the lower spatial resolution of

10 GCMs in comparison to the regional model, SMB is underestimated at the coasts while an overestimation occurs in the interior of the ice sheet. The bias in the difference between the coastal and higher elevation regions are smaller for the models that have a higher spatial resolution, such as CCSM4 (Fig. S10), confirming that the spatial resolution has a crucial impact on the simulated SMB. However, models with similar resolutions may also have very different results, in particular in coastal regions (relative SMB biases of $+47 \%$ and $+100 \%$ for CCSM4 and MRI-CGCM3 respectively compared to RACMO for DML coast over the 1979-2005 period), suggesting a critical role of model physics in some of the GCM biases. 

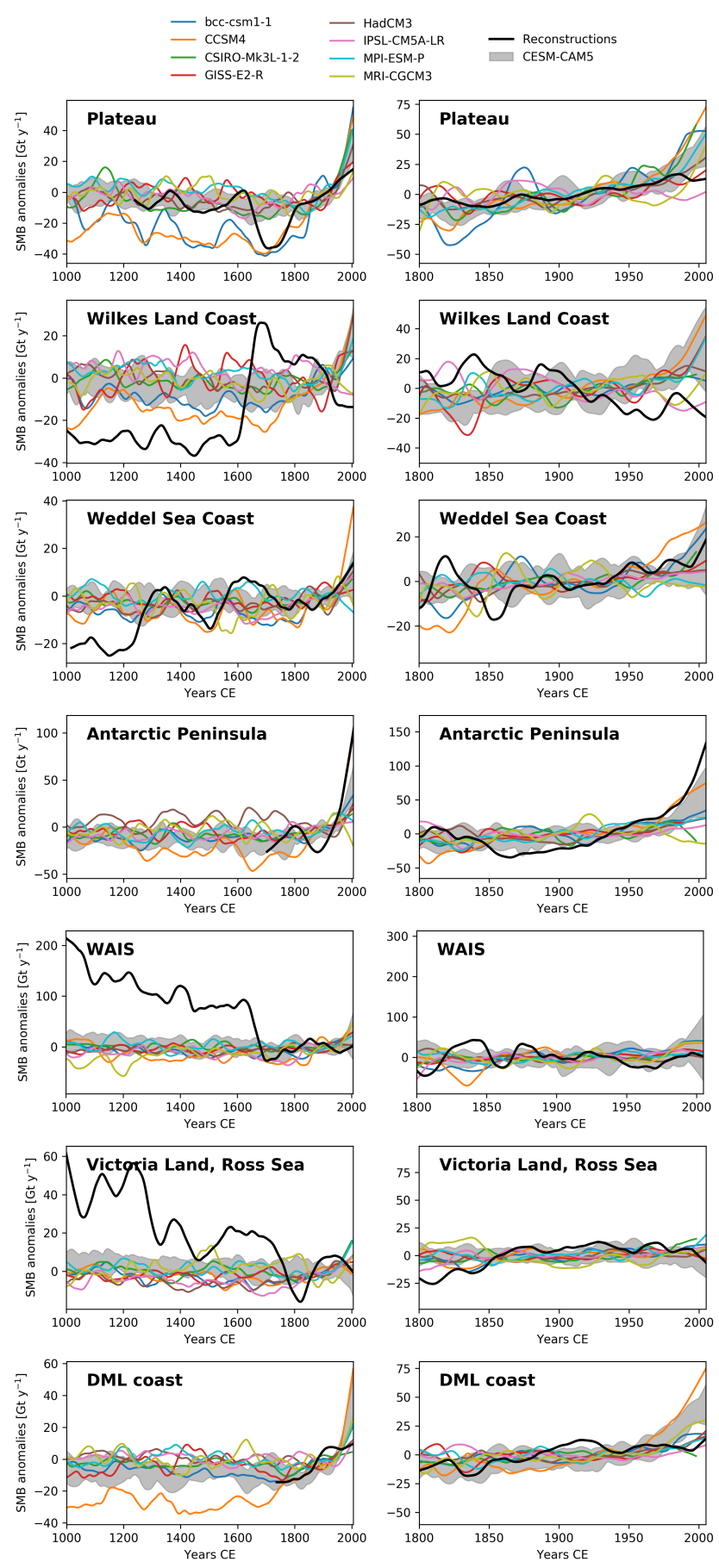

Figure S1. Surface mass balance anomalies $\left[\mathrm{Gt}^{-1}\right]$ simulated by the GCMs (the average of all the available simulations has been represented; Tab. A1) and snow accumulation reconstructions (Thomas et al., 2017) for 1000-2005 and for 1800-2005 for all the Antarctic subregions. Anomalies are computed for the 1800-2000 period. The shaded area corresponds to the range of the CESM1-CAM5 simulations. For visibility, data has been smoothed with a 100 year moving average for the last millennium and a 30 years moving average for the last 200 years. 


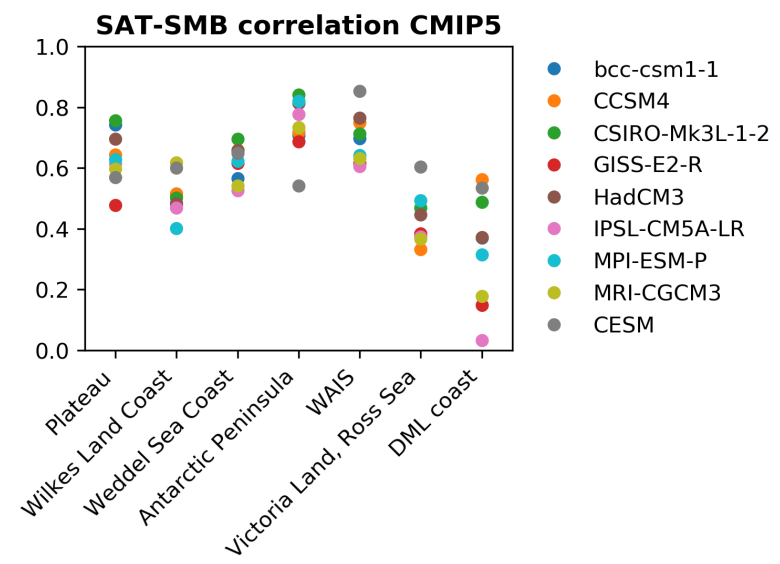

Figure S2. Annual correlations (r) between surface mass balance and surface air temperature for all seven Antarctic regions (see Fig. 1 for geographical definitions) for all the GCMs over the 1850-2005 period.
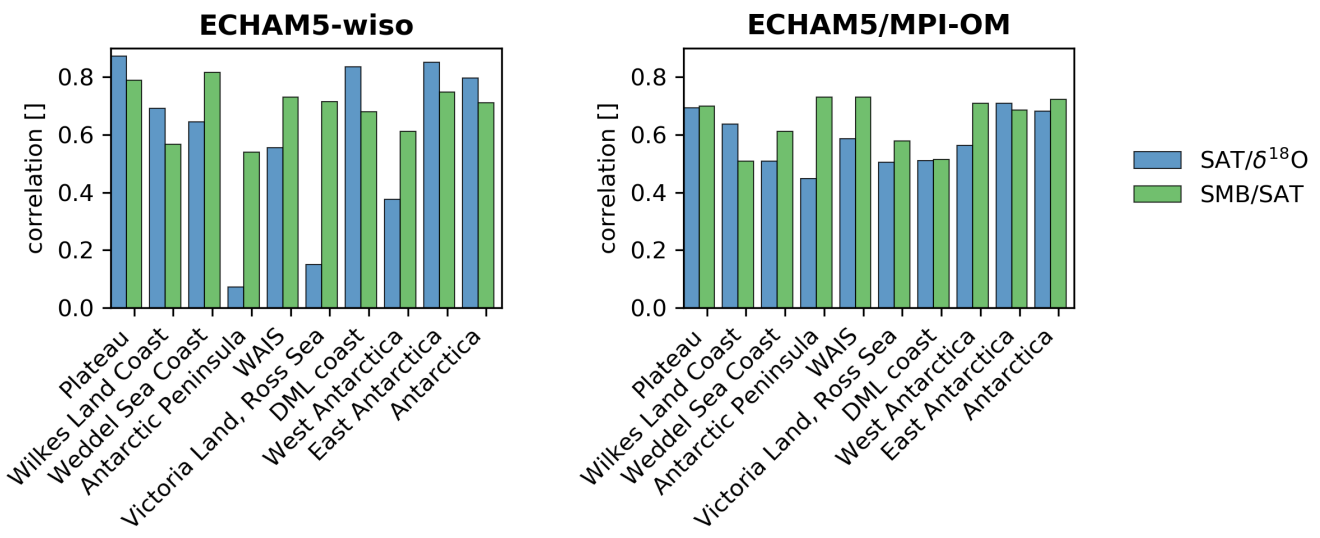

Figure S3. 5-year mean correlations between surface air temperature and $\delta^{18} \mathrm{O}$ (blue) and between SMB and surface air temperature (green) for the seven Antarctic regions for the entire period simulation (1871-2010 for ECHAM5-wiso and 801-2000 for ECHAM5/MPI-OM). 


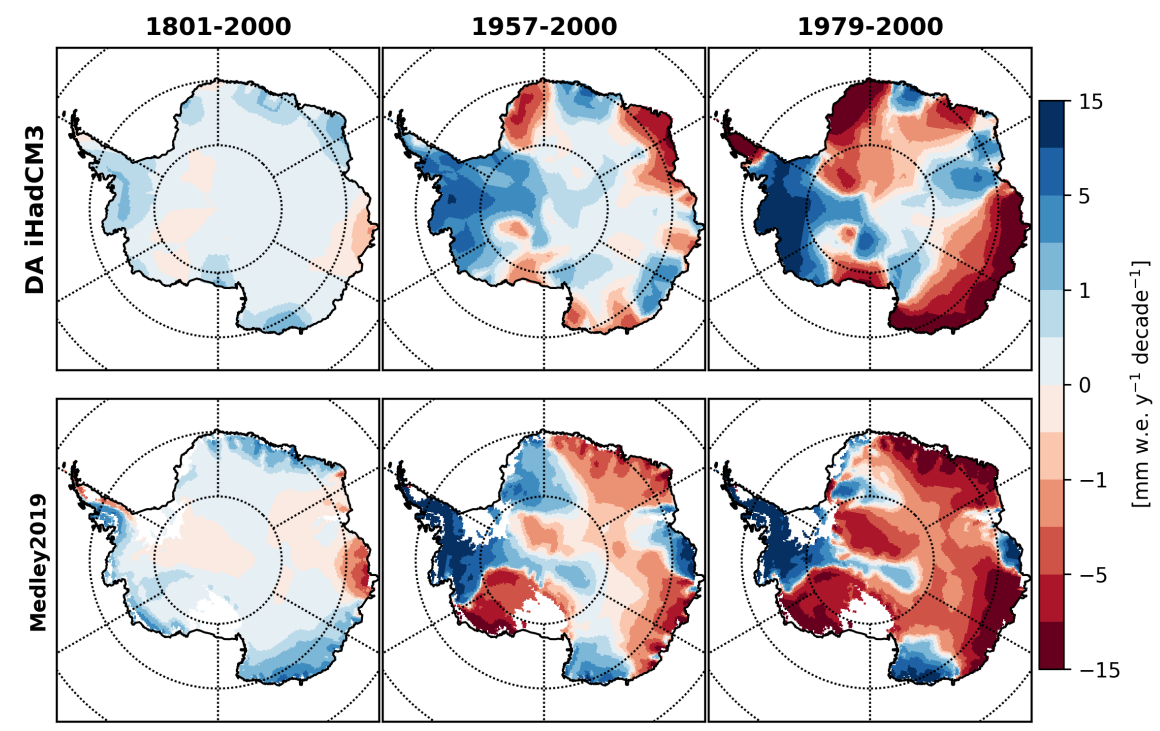

Figure S4. Spatial Antarctic surface mass balance trends (mm w.e. $\mathrm{y}^{-1}$ decade ${ }^{-1}$ ) over the 1801-2000, 1957-2000 and 1979-2000 periods from 1) our data assimilation-based reconstruction using the iHadCM3 outputs constrained by both $\delta^{18} \mathrm{O}$ and SMB (first row) and from 2) Medley and Thomas (2019; second row). 

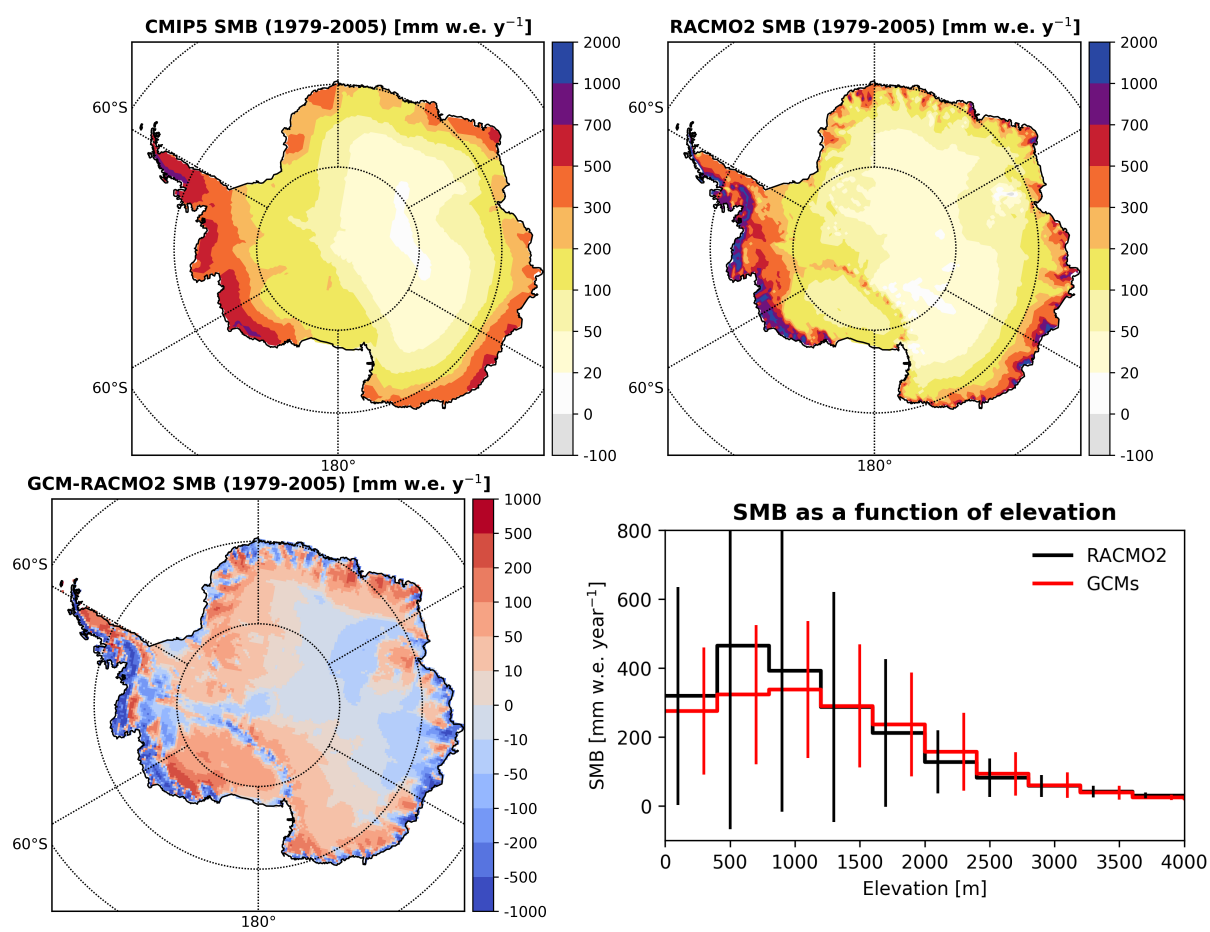

Figure S5. Antarctic Ice Sheet Surface Mass Balance [mm w.e. $\mathrm{y}^{-1}$ ] over 1979-2005 CE averaged over all the GCMs simulations (see Tab. A1 for the list) (top left), for RACMO2 (van Wessem et al., 2018) (top right), the difference between them (bottom left) and the distribution of the SMB simulated by RACMO2 and the GCMs as a function of elevation, binned in 400m elevation intervals (bottom right). The bars represent one standard deviation of the cell grids within each elevation bin. The equivalent of the latter panel for each model is provided on Fig. S10. 


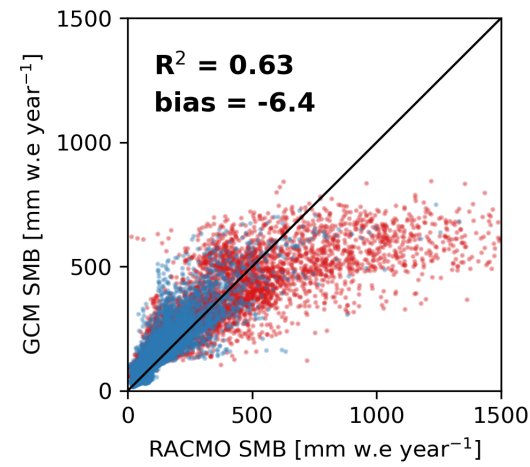

Figure S6. Correlation plot of SMB climatology from GCM mean (average over all the GCMs including isotope-enabled models) as a function of RACMO SMB over the 1979-2005 period. $\mathrm{R}^{2}$ is the determination coefficient and bias the average of the difference between GCM mean and RACMO (in mm w.e. year ${ }^{-1}$ ). Red (blue) dots are for places where the altitude is lower (higher) than $1500 \mathrm{~m}$. See Fig. S8 for each model. 

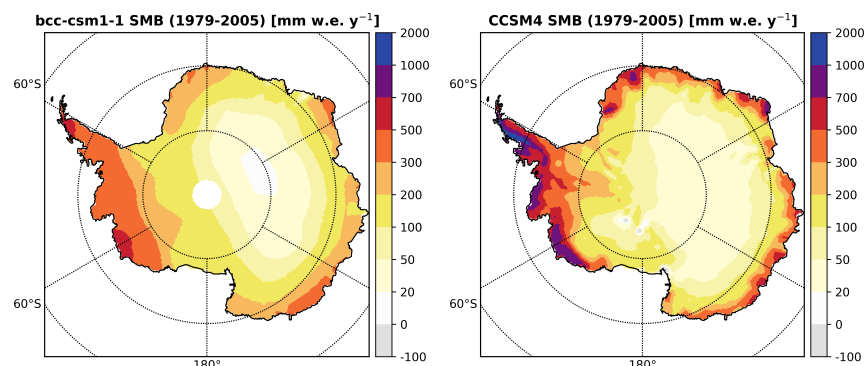

CSIRO-MK3L-1-2 SMB (1979-2005) [mm w.e. $\left.\mathrm{y}^{-1}\right]$
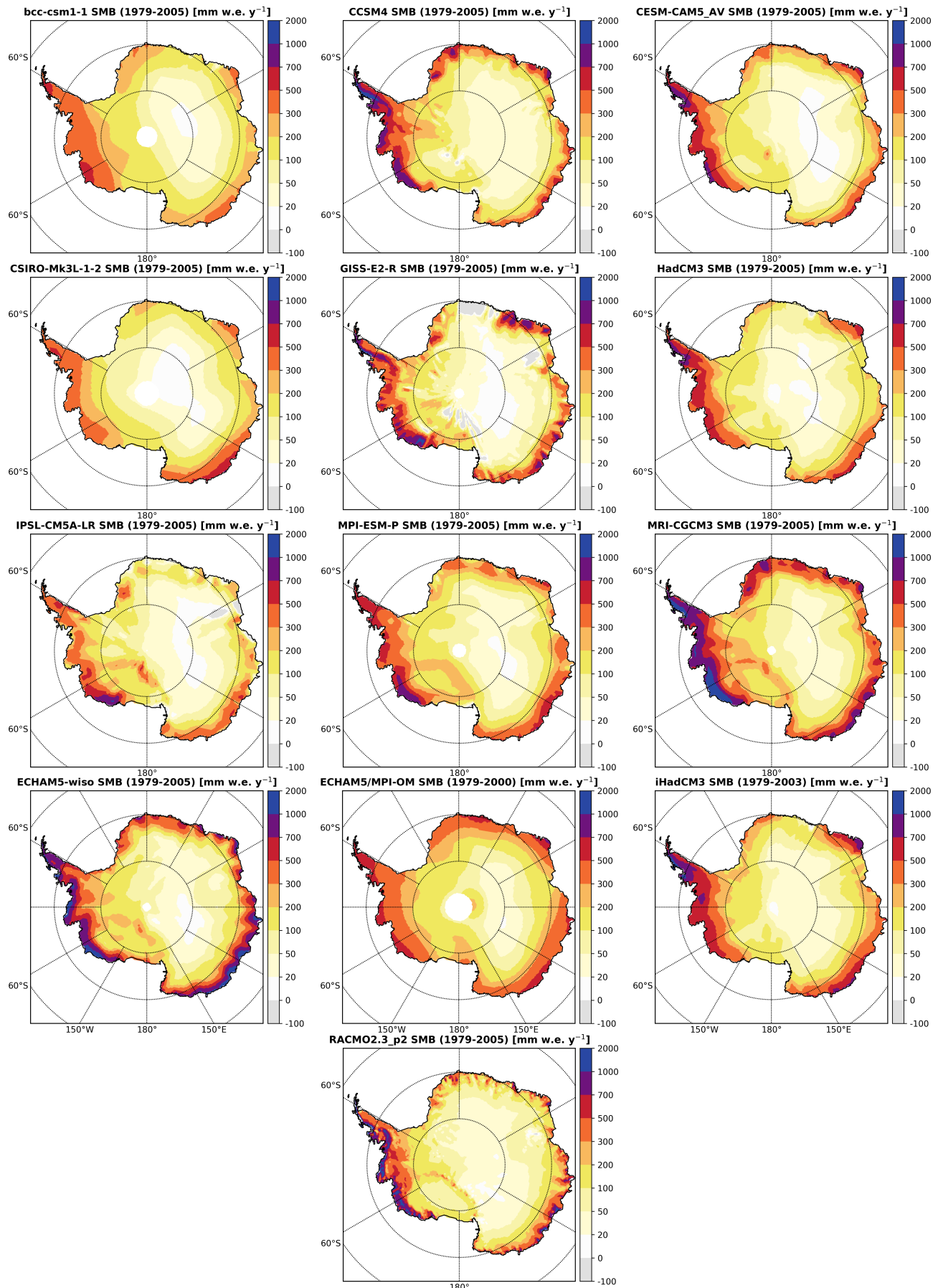

Figure S7. Antarctic Ice Sheet surface mass balance [mm w.e. $\mathrm{y}^{-1}$ ] for all the models used in this study over the 1979-2005 period. 

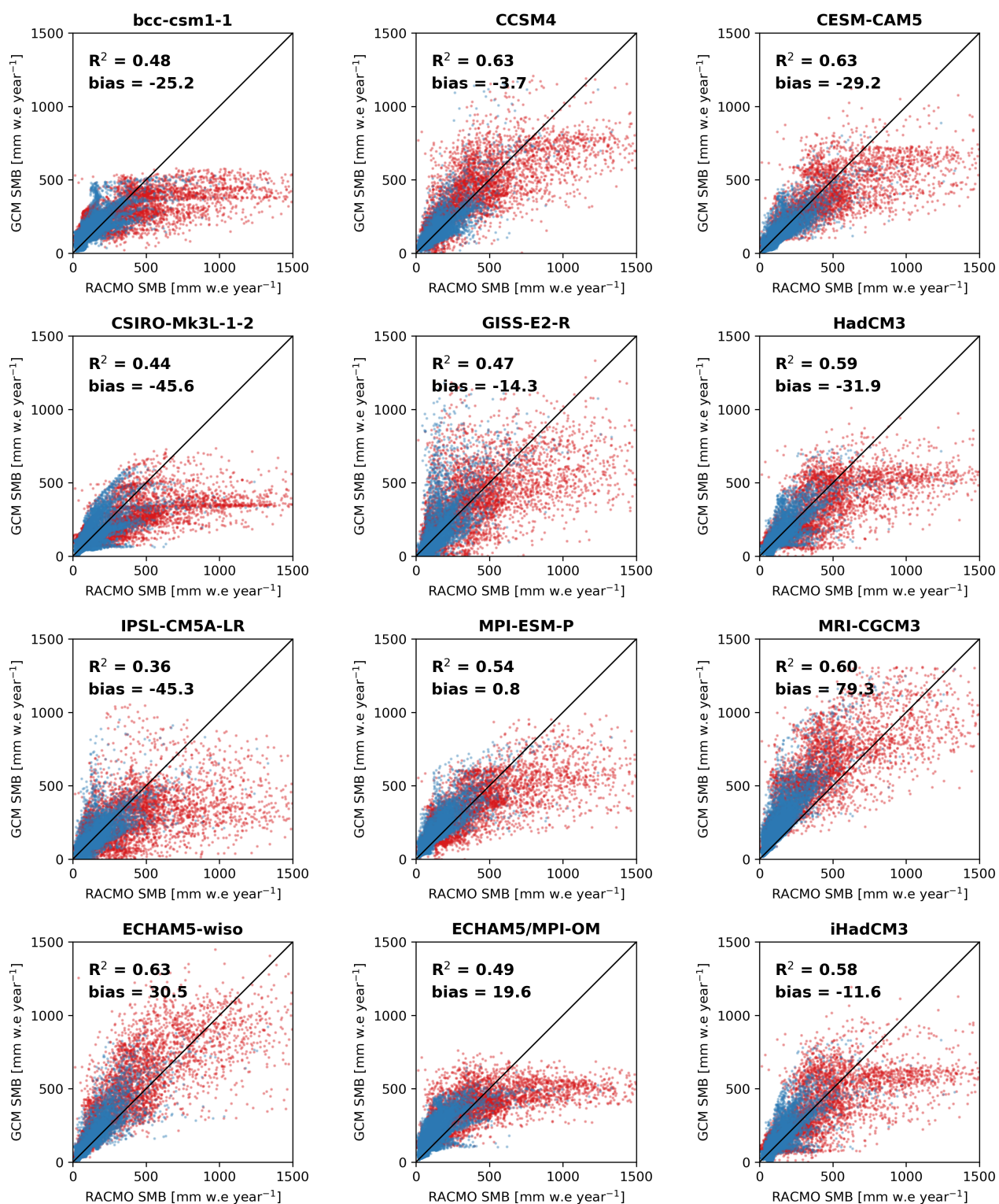

Figure S8. As in Fig. S6 but for all GCMs. 


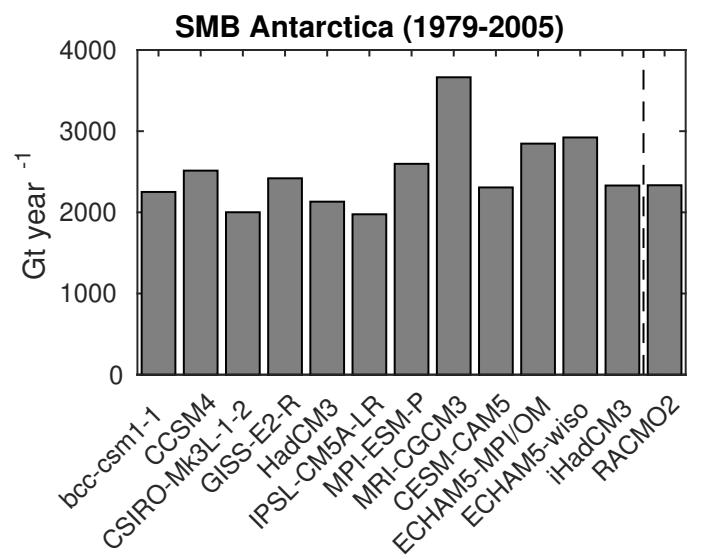

Figure S9. Mean Antarctic Ice Sheet surface mass balance (Gt year $\left.{ }^{-1}\right)$ simulated by all the models used in this study. 

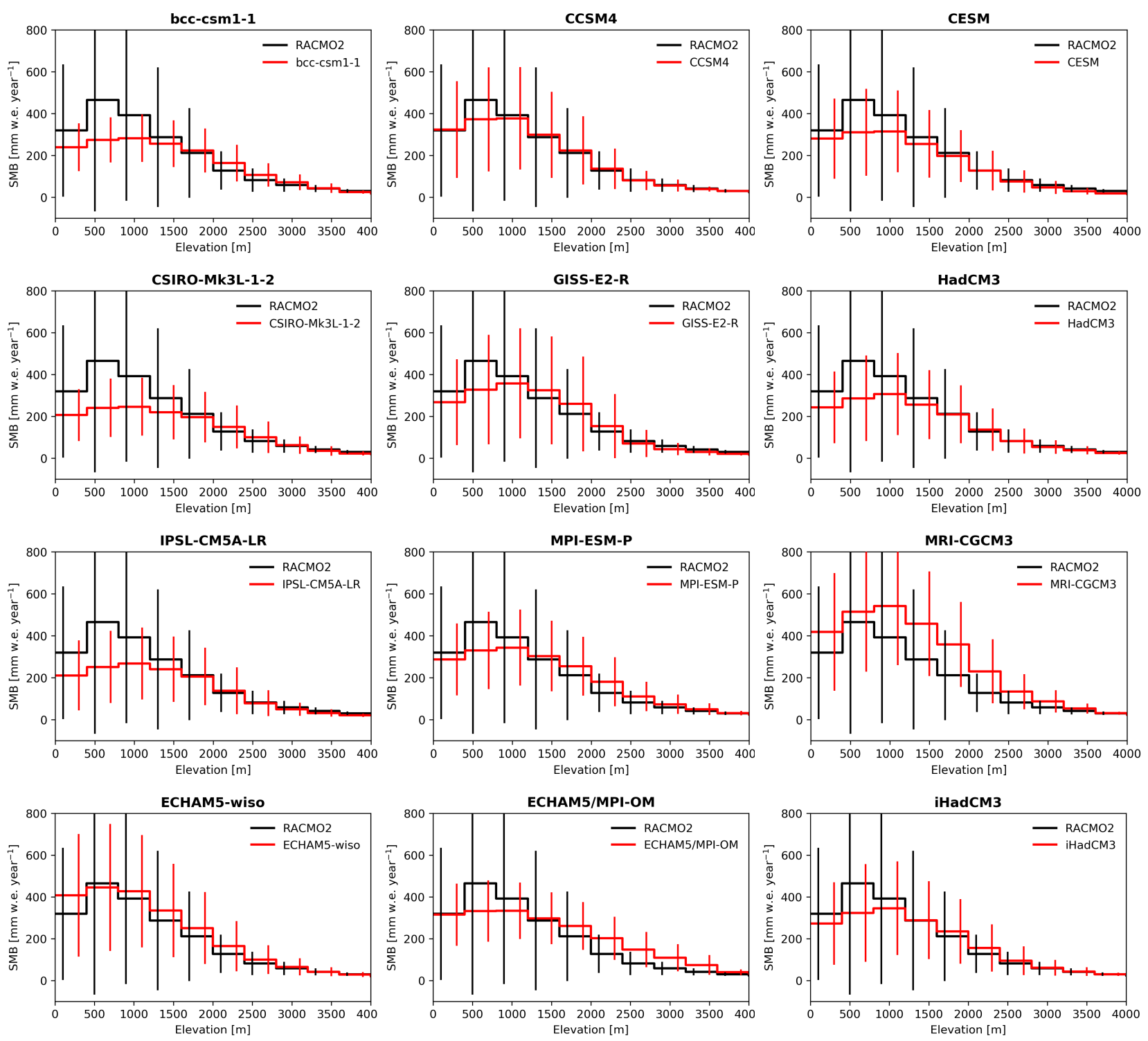

Figure S10. Distribution of the surface mass balance simulated by all climate models used in this study as a function of elevation, binned in $400 \mathrm{~m}$ elevation intervals. The bars represent one standard deviation of the cell grids within each elevation bin. 

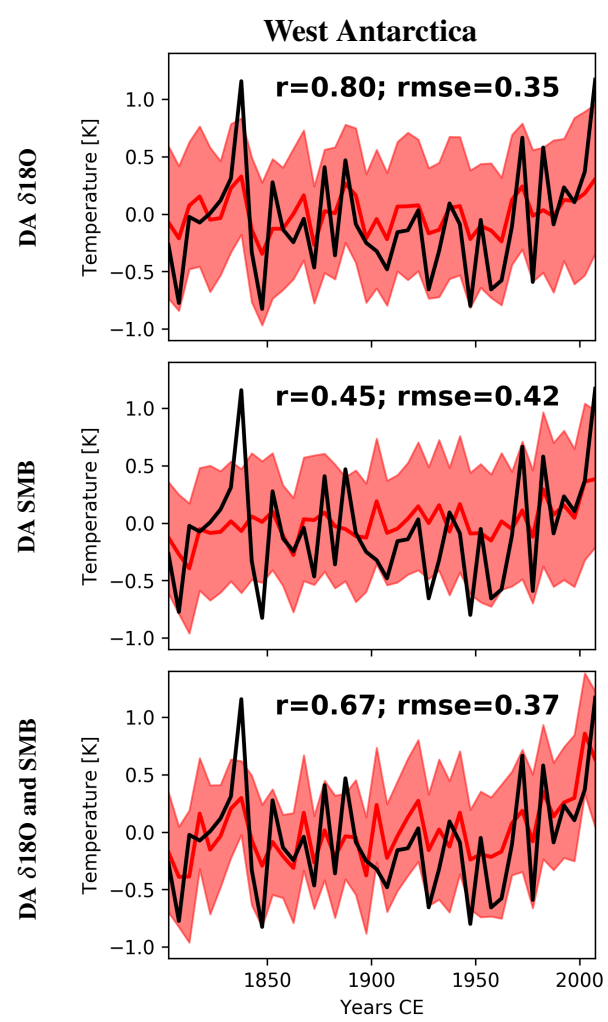

East Antarctica
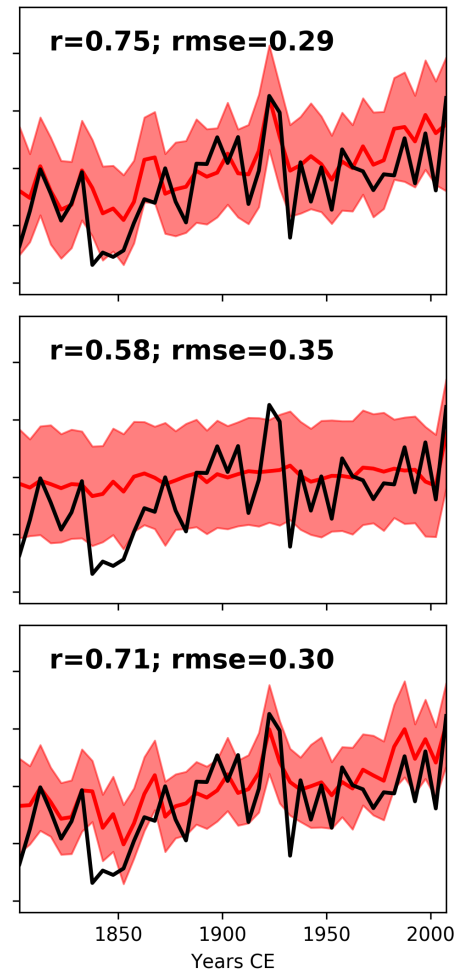

Antarctica
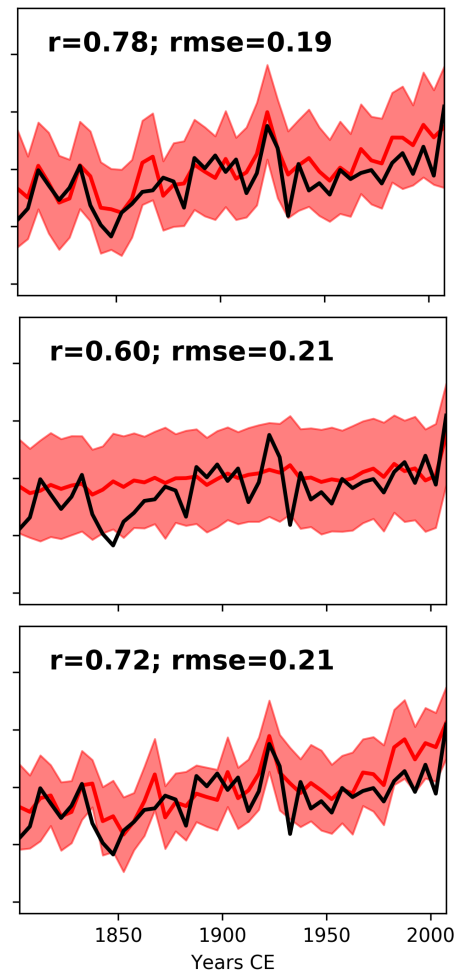

Figure S11. Reconstructed surface air temperatures (5-year mean) for West Antarctica, East Antarctica and Antarctica as a whole from our data assimilation experiment using the ECHAM5-wiso outputs and, $\delta^{18} 0$ (Stenni et al., 2017) and SMB reconstruction (Thomas et al., 2017) as data. The period is $1800-2010 . D A \delta^{18} O$ is the data assimilation experiment using only the $\delta^{18} \mathrm{O}$ data to constrain the model while $D A$ $S M B$ uses only the SMB reconstruction and $D A \delta^{18} O$ and $S M B$ uses both. For each experiment and each region, the correlation $(r)$ between the reconstruction based on ice cores and that based on data assimilation is computed. The shaded areas represent \pm 1 standard deviation of the model particles. 

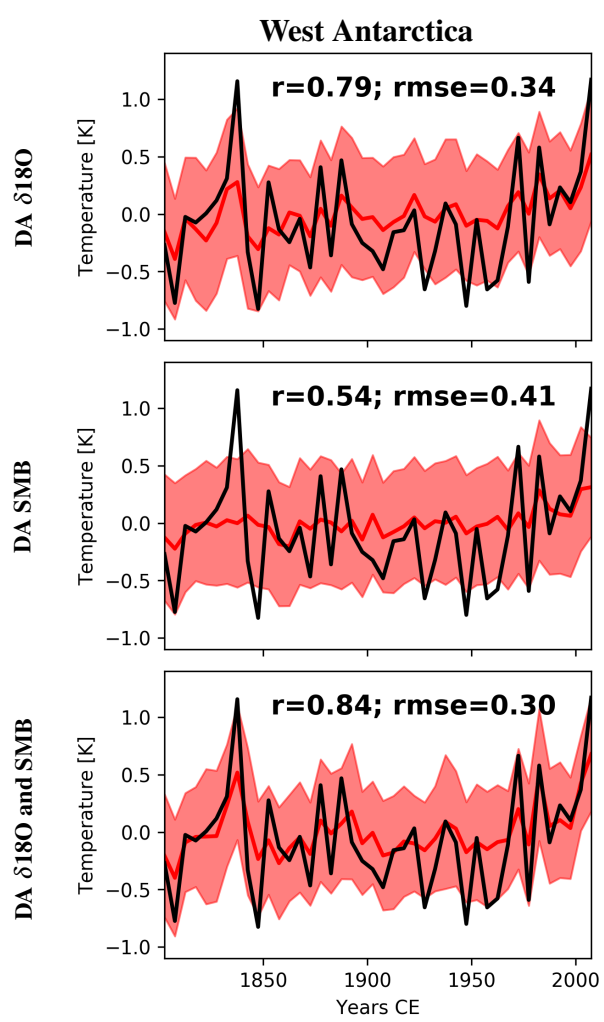

East Antarctica
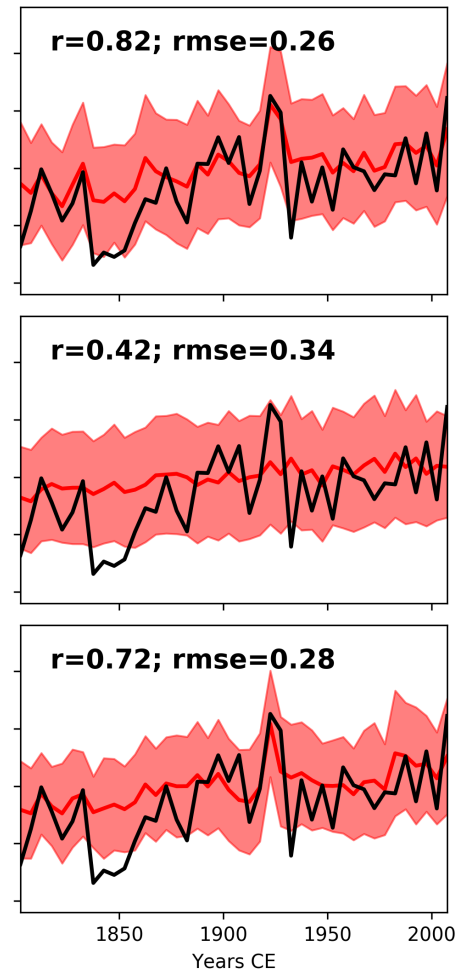

Antarctica

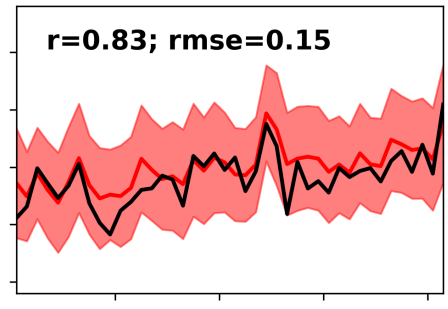

$\mathbf{r}=0.47 ; \mathrm{rmse}=0.20$
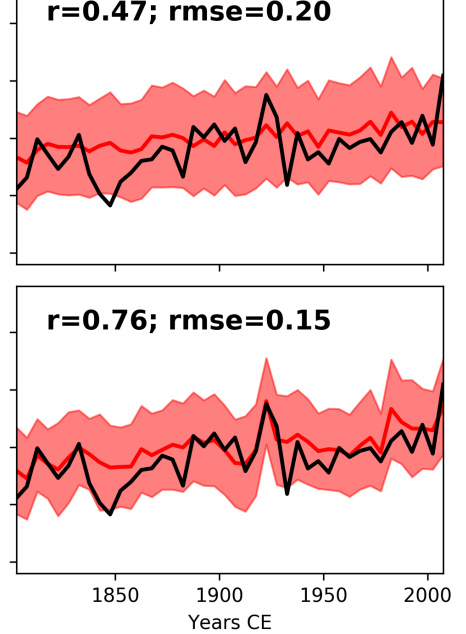

Figure S12. Reconstructed surface air temperatures (5-year mean) for West Antarctica, East Antarctica and Antarctica as a whole from data assimilation experiment using the ECHAM5-MPI/OM outputs and, $\delta^{18} \mathrm{O}$ (Stenni et al., 2017) and SMB reconstruction (Thomas et al., 2017) as data. The period is $1800-2010 . D A \delta^{18} O$ is the data assimilation experiment using only the $\delta^{18} \mathrm{O}$ data to constrain the model while $D A$ $S M B$ uses only the SMB reconstruction and $D A \delta^{18} O$ and $S M B$ uses both. For each experiment and each region, the correlation $(r)$ between the reconstruction based on ice cores and that based on data assimilation is computed. The shaded areas represent \pm 1 standard deviation of the model particles. 

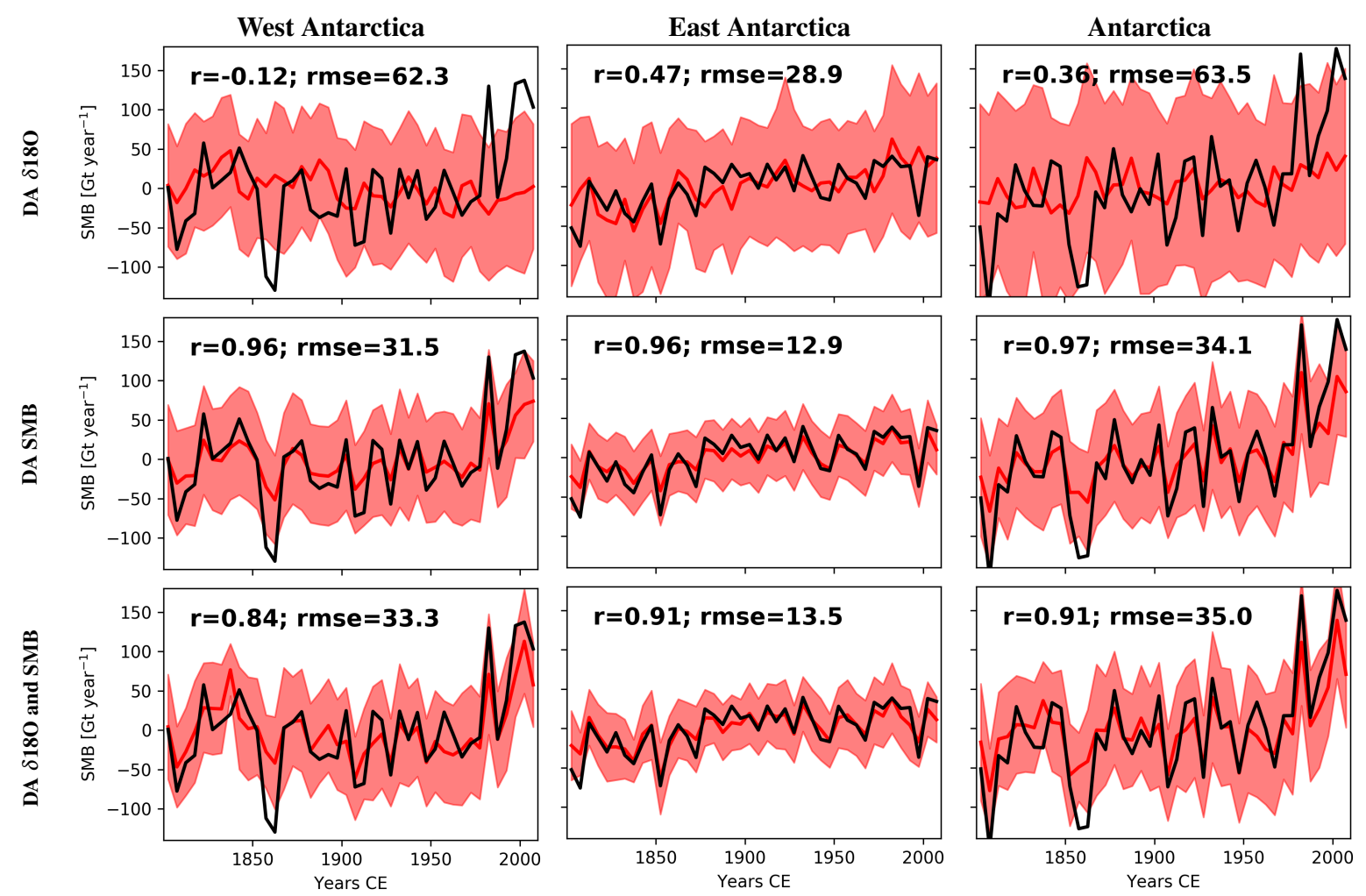

Figure S13. Reconstructed SMB (5-year mean) for West Antarctica, East Antarctica and Antarctica as a whole from data assimilation experiment using the ECHAM5-wiso outputs and, $\delta^{18} \mathrm{O}$ (Stenni et al., 2017) and SMB reconstruction (Thomas et al., 2017) as data. The period is $1800-2010 . D A \delta^{18} O$ is the data assimilation experiment using only the $\delta^{18} \mathrm{O}$ data to constrain the model while $D A S M B$ uses only the SMB reconstruction and $D A \delta^{18} O$ and $S M B$ uses both. For each experiment and each region, the correlation $(r)$ between the reconstruction based on ice cores and that based on data assimilation is computed. The shaded areas represent \pm 1 standard deviation of the model particles. 

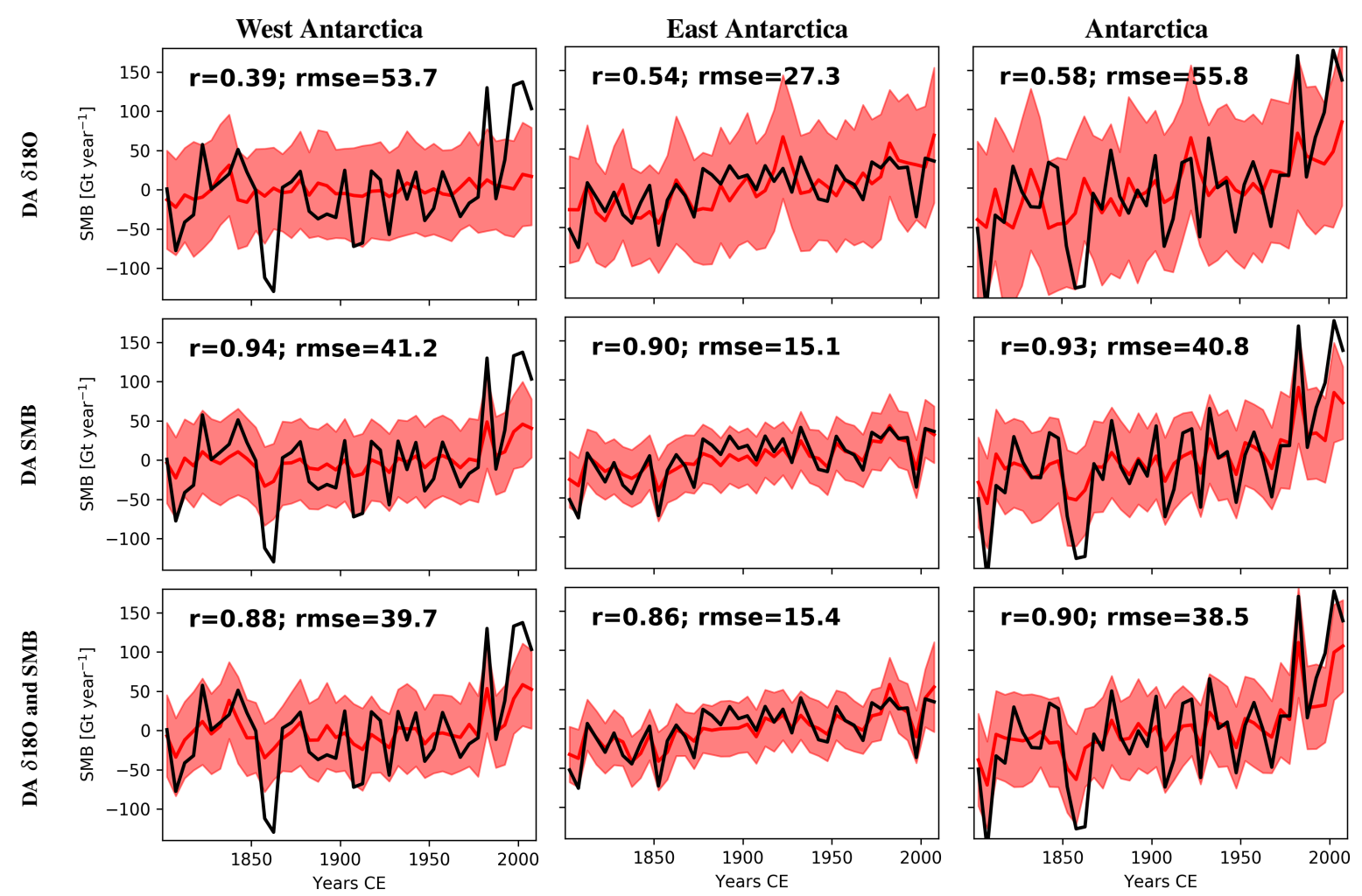

Figure S14. Reconstructed SMB (5-year mean) for West Antarctica, East Antarctica and Antarctica as a whole from data assimilation experiment using the ECHAM5-MPI/OM outputs and, $\delta^{18} \mathrm{O}$ (Stenni et al., 2017) and SMB reconstruction (Thomas et al., 2017) as data. The period is $1800-2010 . D A \delta^{18} O$ is the data assimilation experiment using only the $\delta^{18} \mathrm{O}$ data to constrain the model while $D A S M B$ uses only the SMB reconstruction and $D A \delta^{18} O$ and $S M B$ uses both. For each experiment and each region, the correlation $(r)$ between the reconstruction based on ice cores and that based on data assimilation is computed. The shaded areas represent \pm 1 standard deviation of the model particles. 
Table S1. Surface mass balance trends (in Gt $100 \mathrm{y}^{-2}$ ) for West Antarctica, East Antarctica and Antarctica as a whole in GCMs, in isotopic climate models (ECHAM5-wiso, ECHAM5/MPIOM and HadCM3) and in reconstructions based on ice cores (Thomas et al., 2017) over 1950-2000. The number in brackets is the number of simulations. The trend computation is based on yearly data.

\begin{tabular}{|c|c|c|c|c|c|c|c|c|c|}
\hline & \multicolumn{3}{|c|}{ West Antarctica } & \multicolumn{3}{|c|}{ East Antarctica } & \multicolumn{3}{|c|}{ Antarctica } \\
\hline & $\min$ & $\max$ & mean & $\min$ & $\max$ & mean & $\min$ & $\max$ & mean \\
\hline bcc-csm1-1 (3) & -29.46 & 152.47 & 77.62 & -63.11 & 381.09 & 200.39 & -92.57 & 533.56 & 278.01 \\
\hline CCSM4 (6) & 148.02 & 390.50 & 234.13 & 274.32 & 455.65 & 368.19 & 469.24 & 846.15 & 602.32 \\
\hline CSIRO-Mk3L-1-2 (1) & & & 3.14 & & & 135.86 & & & 139.00 \\
\hline GISS-E2-R (6) & 25.69 & 183.66 & 107.27 & -71.92 & 250.18 & 140.43 & -46.23 & 416.21 & 247.71 \\
\hline HadCM3 (10) & 4.79 & 150.27 & 70.75 & -68.85 & 242.18 & 89.39 & -34.18 & 303.11 & 160.14 \\
\hline IPSL-CM5A-LR (6) & 57.07 & 123.78 & 99.07 & -104.18 & 66.82 & -10.06 & -47.11 & 174.30 & 89.01 \\
\hline MPI-ESM-P (2) & -33.85 & -28.74 & -31.30 & 54.75 & 231.84 & 143.29 & 26.01 & 197.99 & 112.00 \\
\hline MRI-CGCM3 (3) & 28.62 & 178.64 & 86.45 & -59.28 & 242.24 & 125.66 & -7.19 & 420.89 & 212.11 \\
\hline CESM1-CAM5 (12) & 30.90 & 349.67 & 153.07 & 55.72 & 340.24 & 162.27 & 161.99 & 592.43 & 315.34 \\
\hline iHadCM3 (6) & 76.23 & 232.69 & 162.29 & 15.52 & 350.87 & 213.61 & 115.85 & 542.61 & 375.90 \\
\hline ECHAM5-wiso (1) & & & -8.79 & & & 195.22 & & & 186.43 \\
\hline ECHAM5/MPIOM (1) & & & 41.44 & & & 35.43 & & & 76.87 \\
\hline Reconstructions (1) & & & 256.74 & & & -35.80 & & & 220.95 \\
\hline
\end{tabular}

Table S2. 5-year mean correlations between the three surface air temperature reconstructions from data assimilation experiments using the iHadCM3 outputs and the statistical reconstruction of Stenni et al. (2017), with the surface air temperature reconstructions from Nicolas and Bromwich (2014) over the 1958-2010 period for East Antarctica, West Antarctica and Antarctica as a whole. All the correlations are performed on detrended time series. Stars represent statistically significant correlations ( $\mathrm{p}$-value<0.10).

\begin{tabular}{lccc}
\hline & West Antarctica & East Antarctica & Antarctica \\
\hline DA $\delta^{18} \mathrm{O}$ & -0.02 & -0.16 & -0.25 \\
DA SMB & -0.19 & 0.51 & 0.31 \\
DA $\delta^{18} \mathrm{O}$ and SMB & 0 & $0.60^{*}$ & 0.44 \\
\hline Stenni et al. (2017) & $0.45^{*}$ & -0.20 & $0.12^{*}$ \\
\hline
\end{tabular}


Table S3. Slopes $\left({ }^{\circ} \mathrm{C} 100 \mathrm{yr}^{-1}\right)$ of each surface air temperature reconstruction (Stenni et al., 2017; Klein et al., 2019; Nicolas and Bromwich, 2014; in this study) over the 1961-2010 period for West Antarctica, East Antarctica and the Antarctica. Statistically significant (p-value < $0.05)$ trends are represented by a star.

\begin{tabular}{|c|c|c|c|}
\hline Dataset & $\begin{array}{c}\text { West } \\
\text { Antarctica }\end{array}$ & $\begin{array}{c}\text { East } \\
\text { Antarctica }\end{array}$ & Antarctica \\
\hline \multicolumn{4}{|l|}{ Stenni et al. (2017) } \\
\hline Stat ECHAMvariance & $1.69^{*}$ & $0.75^{*}$ & 1.27 \\
\hline Stat borehole & $2.07 *$ & $0.75^{*}$ & $0.77 *$ \\
\hline \multicolumn{4}{|l|}{ Klein et al. (2018) } \\
\hline DA ECHAM5-wiso & 1.15 & 0.94 & 0.98 \\
\hline DA ECHAM5/MPI-OM & 1.0 & 0.48 & 0.59 \\
\hline \multicolumn{4}{|l|}{ Nicolas and Bromwich (2014) } \\
\hline & $2.22 *$ & 0.53 & $0.90^{*}$ \\
\hline \multicolumn{4}{|l|}{ In this study } \\
\hline DA $\delta^{18} \mathrm{O}$ and SMB iHadCM3 & $0.99 *$ & $0.60 *$ & $0.69^{*}$ \\
\hline
\end{tabular}

